\title{
In Vivo Confocal Microscopy of Meibomian Glands in Sjögren's Syndrome
}

\author{
Edoardo Villani, Silvia Beretta, Michela De Capitani, Daniela Galimberti, Francesco Viola, \\ and Roberto Ratiglia
}

Purpose. To evaluate morphologic changes in meibomian glands (MGs) and the status of periglandular inflammation in patients with primary and secondary Sjögren's Syndrome (SS) using in vivo confocal laser microscopy (LSCM).

METHODS. Twenty patients with primary SS (SSI), 25 with secondary SS (SSII), 20 with MG dysfunction (MGD), and 25 age- and gender-matched control subjects were enrolled consecutively. Each participant completed an Ocular Surface Disease Index questionnaire and underwent a full eye examination, including tear film break-up time (BUT), fluorescein and lissamine green staining, Schirmer test, and an LSCM examination of the MGs, the last to determine acinar unit density and diameter, glandular orifice diameters, meibum secretion reflectivity, inhomogeneous appearance of glandular interstice, and acinar wall.

RESULTs. All parameters indicated statistically significant differences among groups ( $P<0.001$, Kruskal-Wallis test). LSCM demonstrated no differences between SSI and SSII (MannWhitney $U$ test). Compared with control subjects, SS subjects MGs showed more periglandular inflammation and higher secretion reflectivity ( $P<0.001$, Mann-Whitney $U$ test). Compared with MGD patients, SS patients' MGs had higher acinar density, smaller diameters, greater density of periglandular inflammatory cells, and lower secretion reflectivity $(P<0.001$, Mann-Whitney $U$ test). In SS patients, the two measured confocal signs of inflammation were significantly interrelated and correlated with corneal fluorescein staining $(P \leq 0.01$, Spearman correlation coefficient). Acinar density and diameters were strongly correlated among themselves $(P<0.001)$ and with BUT $(P<0.05)$.

Conclusions. LSCM is capable of effectively revealing morphologic and inflammatory changes in MGs and showed discernible patterns of MG abnormalities in SS and MGD not easily distinguishable by the usual clinical exams. (Invest Ophthalmol Vis Sci. 2011;52:933-939) DOI:10.1167/iovs.10-5995

Sjögren's Syndrome (SS), a relatively common chronic autoimmune disorder affecting $\sim 1 \%-2 \%$ of the population, is characterized by dysfunction and destruction of exocrine glands - mainly salivary and lacrimal glands. SS can be found alone-primary SS (SSI) - or accompanied by almost any other

From the ${ }^{1}$ Università degli Studi di Milano, UO Oculistica Fondazione IRCCS Cà Granda Ospedale Maggiore Policlinico, Milan, Italy.

Submitted for publication June 3, 2010; revised August 16, 2010 ; accepted September 4, 2010.

Disclosure: E. Villani, None; S. Beretta, None; M. De Capitani, None; D. Galimberti, None; F. Viola, None; R. Ratiglia, None

Presented at the annual meeting of the Association for Research in Vision and Ophthalmology, Fort Lauderdale, Florida, May 2010.

Corresponding author: Edoardo Villani, Università degli Studi di Milano, UO Oculistica Fondazione IRCCS Cà Granda Ospedale Maggiore Policlinico, Via Francesco Sforza 35, 20122 Milan, Italy; eddy.villani@tiscali.it. autoimmune disease-secondary SS (SSII), wherein a broad spectrum of clinical symptoms that extend from the exocrine glands (organ-specific exocrinopathy) to extraglandular manifestations (systemic disease) can be observed. ${ }^{1}$

Epithelial cells are thought to play important pathogenetic roles, as suggested by the occurrence of infiltrating lesions in various epithelial tissues (described as autoimmune epitheliitis) as well as increased epithelial expression of several inflammatory proteins in the lesions of patients. Epithelial cells in SS are activated and are capable of acting as antigen-presenting cells. $^{2-4}$

SS is classically characterized by oral (xerostomia) and ocular dryness, keratoconjunctivitis sicca (KCS). For many years, KCS associated with SS was considered to be the result of reduced secretion of the aqueous component in tears. ${ }^{5}$ Today, other pathogenetic elements, such as inflammation, changes in tear osmolarity, and alterations in corneal sensitivity and innervation, are considered to be fundamental. These elements interact in a complex (although, as yet, not completely decoded) manner within the ocular surface morpho-functional unit that comprises the tear film, cornea, limbus, conjunctiva, mucocutaneous junction, and lacrimal (principal and accessory) and meibomian glands (MGs). 5,6

MGs are holocrine, lipid-excreting glands embedded in the tarsal plate of the upper and lower lids. Each MG comprises multiple acini that are connected by a long common central duct running throughout the entire length of the gland. MG dysfunction (MGD) is a term primarily used to describe obstructive MG disease. MGD, a major cause of evaporative dry eye, causes alteration and reduction of lipid secretions, leading to increased tear evaporation, decreased tear stability, loss of lubrication, and damage to the ocular surface epithelia. ${ }^{6-8}$ Previous studies have also shown abnormal evaporation rates and clinically significant destruction of MGs in patients with "hyposecretive" KCS associated with SS. ${ }^{9,10}$

In vivo confocal laser microscopy (LSCM) is a new emerging noninvasive technology that is useful as a supplementary diagnostic tool for in vivo assessment of the histopathology of many ocular surface disorders, ${ }^{11}$ including in vivo examination of MGs. ${ }^{12-14}$ In this study we evaluated the morphologic changes of MGs and the status of periglandular inflammation in patients with SSI and SSII using LSCM and investigated the correlation between clinical and confocal findings.

\section{Methods}

\section{Patients}

We consecutively enrolled 20 patients with SSI ( 20 women; mean age, $53.3 \pm 20$ years; range, $35-72$ years), 25 patients with SSII ( 23 women and two men; mean age, $53 \pm 11$ years; range, $42-65$ years), 20 patients with MGD (mean age, $52 \pm 10$ years; range, $40-60$ years), and 25 healthy control subjects (18 women and 7 men; mean age, $50 \pm 15$ years; range, $20-80$ years). 


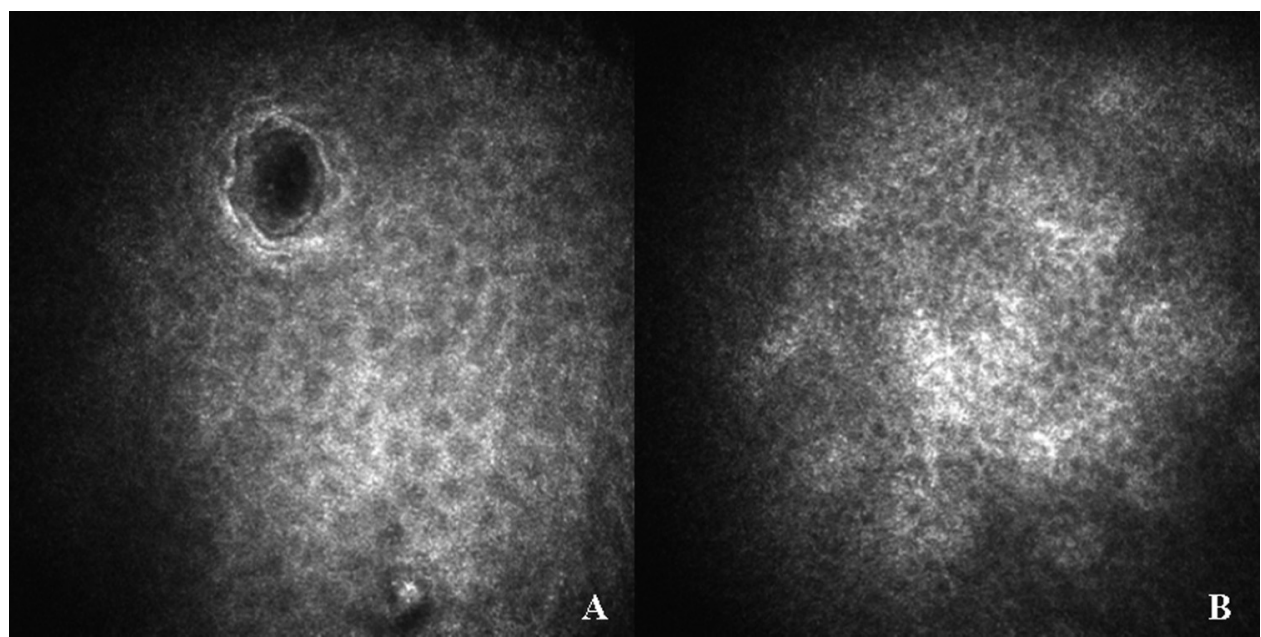

FiguRe 1. (A) Superficial and (B) basal epithelium of the eyelid margin.

Patients with SSI and SSII were recruited at the Referral Centre for Systemic Autoimmune Diseases of Fondazione Cà Granda IRCCS, Ospedale Maggiore Policlinico. The diagnosis was made according to American-European Consensus Group criteria. ${ }^{15}$ MGD and control patients were recruited at the General Clinic of Ophthalmology of Fondazione Cà Granda IRCCS, Ospedale Maggiore Policlinico. The diagnosis of MGD was made according to criteria reported by Matsumoto et al. ${ }^{12}$ Exclusion criteria for all groups included lymphoma, AIDS, diabetes mellitus, contact lens wear, history of eye surgery, and systemic or topical therapy with FAN, steroids, or drugs with known corneal toxicity.
Written informed consent was obtained from all subjects before examination. This study adhered to the tenets of the Declaration of Helsinki.

\section{Clinical Evaluation}

An accurate medical history was recorded for all study participants. All patients completed a questionnaire for standardized evaluation of dry eye-related symptoms: the Ocular Surface Disease Index. All patients underwent a thorough ophthalmic evaluation, including biomicroscopic examination of the ocular adnexa and anterior segment.

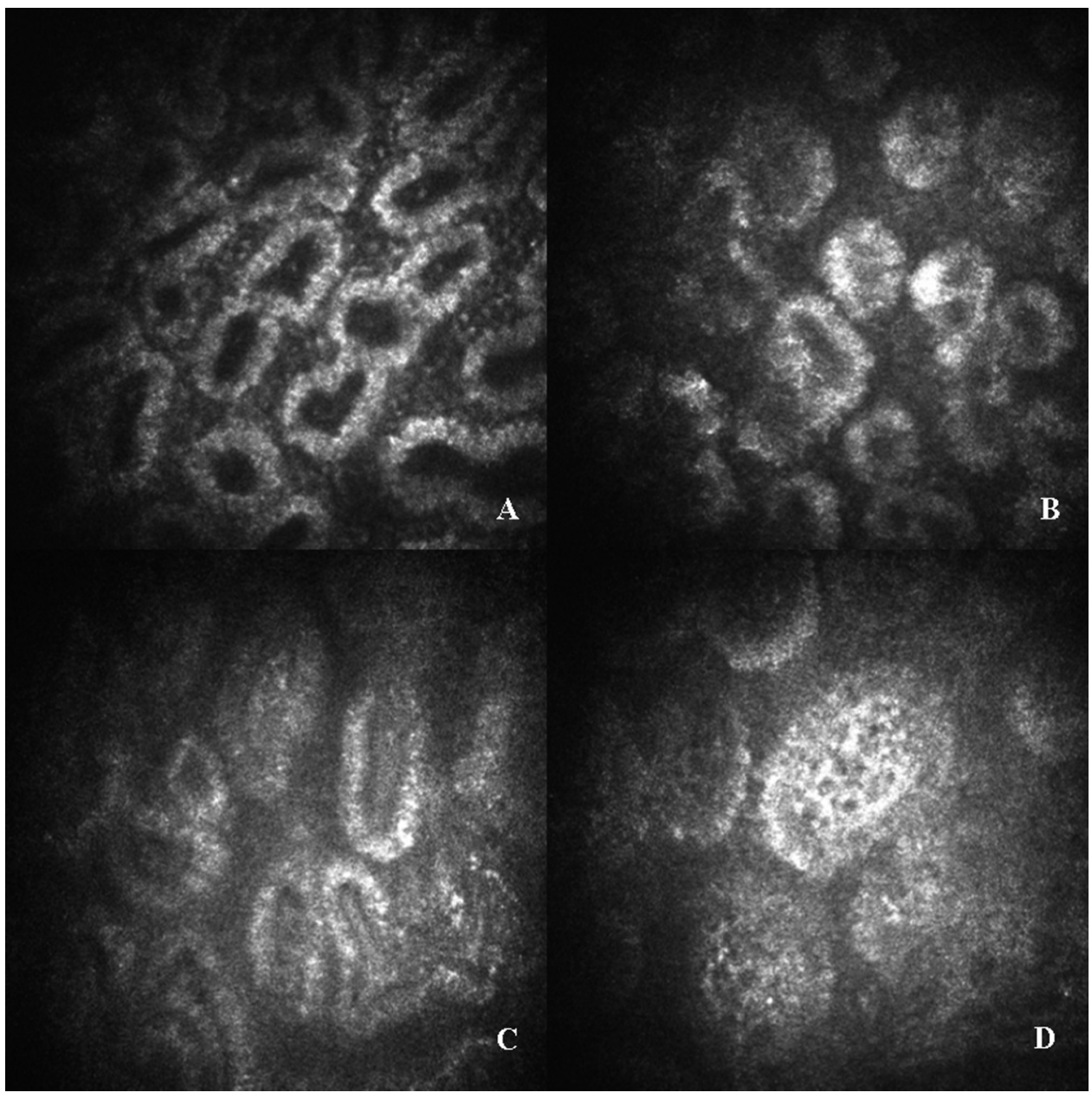

FIGURE 2. Secretion reflectivity in MGs. Grading 1-4: (A) grade 1, black color of secretion; (B) grade 2, dark gray color of secretion; (C) grade 3, light gray color of secretion; (D) grade 4 , white color of secretion. 
To evaluate MGs, transillumination observation (meibography) of the lower eyelid was performed using a fiber-optic device. The degree of MG dropout was scored, as described previously ${ }^{8,10}$ : grade $0=$ no gland dropout; grade 1 = gland dropout in less than half of the inferior tarsus; and grade 2 = gland dropout in more than half of the inferior tarsus.

Tear film break-up time (BUT), corneal staining with fluorescein, and bulbar conjunctival staining with lissamine green were also performed. Ocular surface staining was scored according to the Collaborative Longitudinal Evaluation of Keratoconus scheme. ${ }^{5}$

Tear secretion was evaluated by the Schirmer test using oxybuprocaine chloridrate $0.4 \%$. Assessment of obstruction in MG orifices was conducted by applying digital pressure on the upper tarsus, after which the degree of ease in expressing MG secretion (meibum) was evaluated semiquantitatively: grade $0=$ clear meibum easily expressed; grade $1=$ cloudy meibum expressed with mild pressure; grade $2=$ cloudy meibum expressed with more than moderate pressure; and grade $3=$ meibum not expressed even with firm pressure. 8,10

Both eyes of all subjects were examined. For statistical analysis, the eye with the highest fluorescein staining score was selected. In the case of equal scores for the two eyes, discriminant criteria considered were, in order of relevance, conjunctival staining and BUT.

\section{Confocal Microscopy}

Image Acquisition. LSCM was performed on all subjects using a new-generation confocal microscope (HRT II Corneal Rostock Module; Heidelberg Engineering, Dossenheim, Germany), which employs a $670 \mathrm{~nm}$ scanning wavelength. The objective lens (Zeiss $\times 63$ immer- sion) is covered with a polymethacrylate sterile cap (Tomo-Cap; Heidelberg Engineering) and has a working distance of 0.0-2.0 $\mathrm{mm}$. Before each examination, a drop of oxybuprocaine chlorohydrate $0.4 \%$ and an ophthalmic polyacrylic gel $0.2 \%$ were separately instilled into the conjunctival fornix. After the lower eyelid was partially everted, the center of the sterile cap was applanated onto the center of the eyelid margin (horizontally halfway between the inner and outer canthi), and the instrument focus manually settled (microscope in acquisition modality "Section Mode"), starting from the most superficial down to the deepest discernible tissues having a satisfactory resolution. Ten images were taken every $10 \mu \mathrm{m}$ in depth. Additional images were also taken mid-depth, to manually adjust the quality of the different structures during examination. This procedure was repeated for the nasal and temporal eyelid margins.

Two-dimensional image sizes measured $384 \times 384$ pixels, with a $400 \times 400 \mu \mathrm{m}$ field of view. Each LSCM examination session lasted 3-5 minutes.

Image Analysis. For each variable examined, a single masked investigator (SB) analyzed three randomized, nonoverlapping highquality digital images of the nasal, middle, and temporal lower eyelid margins. We quantified the following variables: cellular density of the superficial and basal epithelia of the eyelid margin (Fig. 1; manually marked inside the largest available region of interest and calculated automatically) (Cell Count software; Heidelberg Engineering), diameters of acinar units (manually measured along the longest axis of the acinar unit), density of MGs (manually marked inside each $400 \times 400 \mu \mathrm{m}$ frame and calculated automatically using Cell Count), ${ }^{12}$ diameter of glandular orifices (manually marked along the longest axis of orifice), meibum secretion reflectivity
FIGURE 3. Inhomogeneous appearance of interstice of acinar unit. Grading 1-4: (A) grade 1, absence of punctate reflecting elements: (B) grade 2 , slight presence of punctate reflecting elements; (C) grade 3, greater presence of punctate reflecting elements; (D) grade 4, higher presence of punctate reflecting elements.

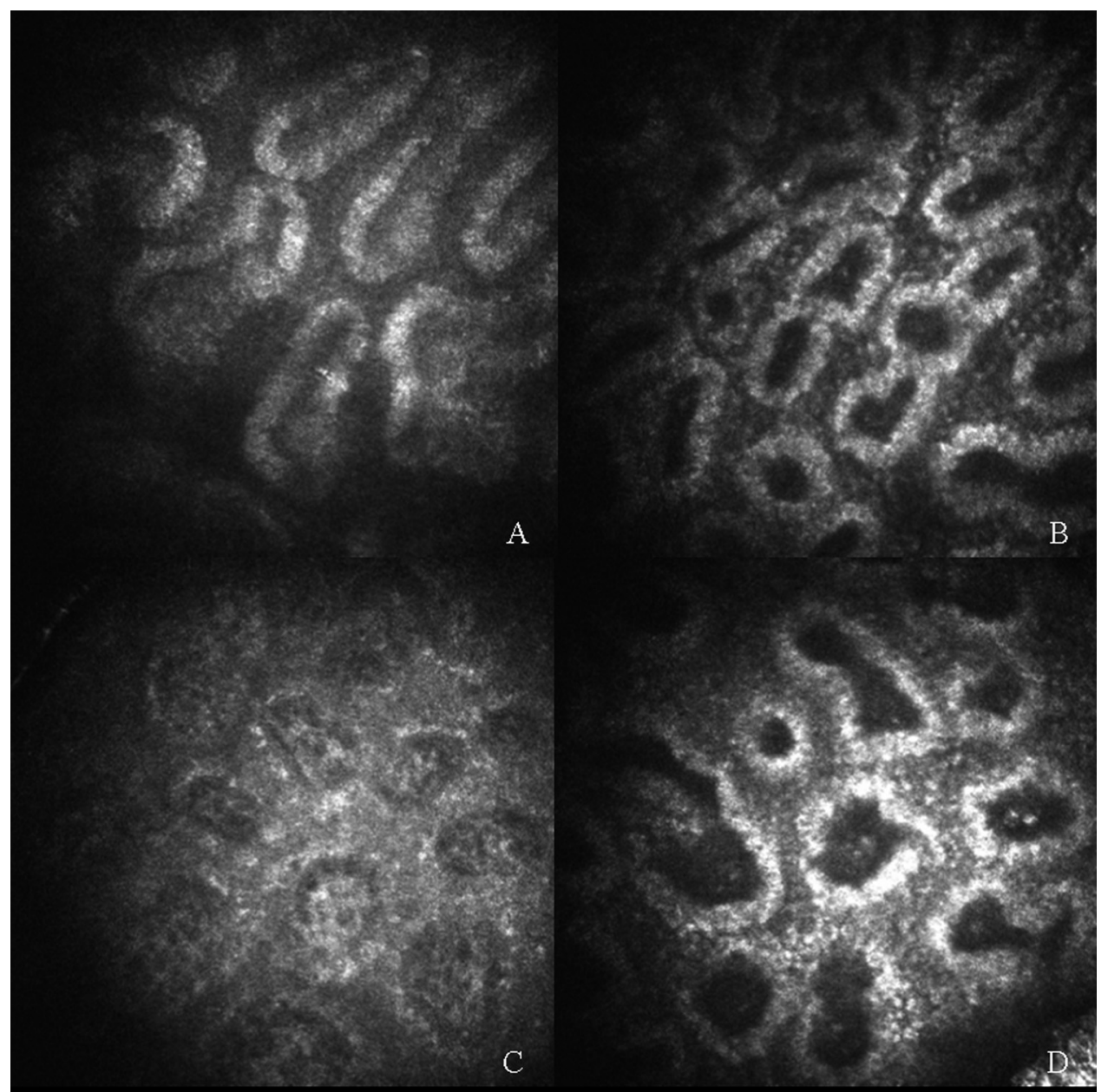




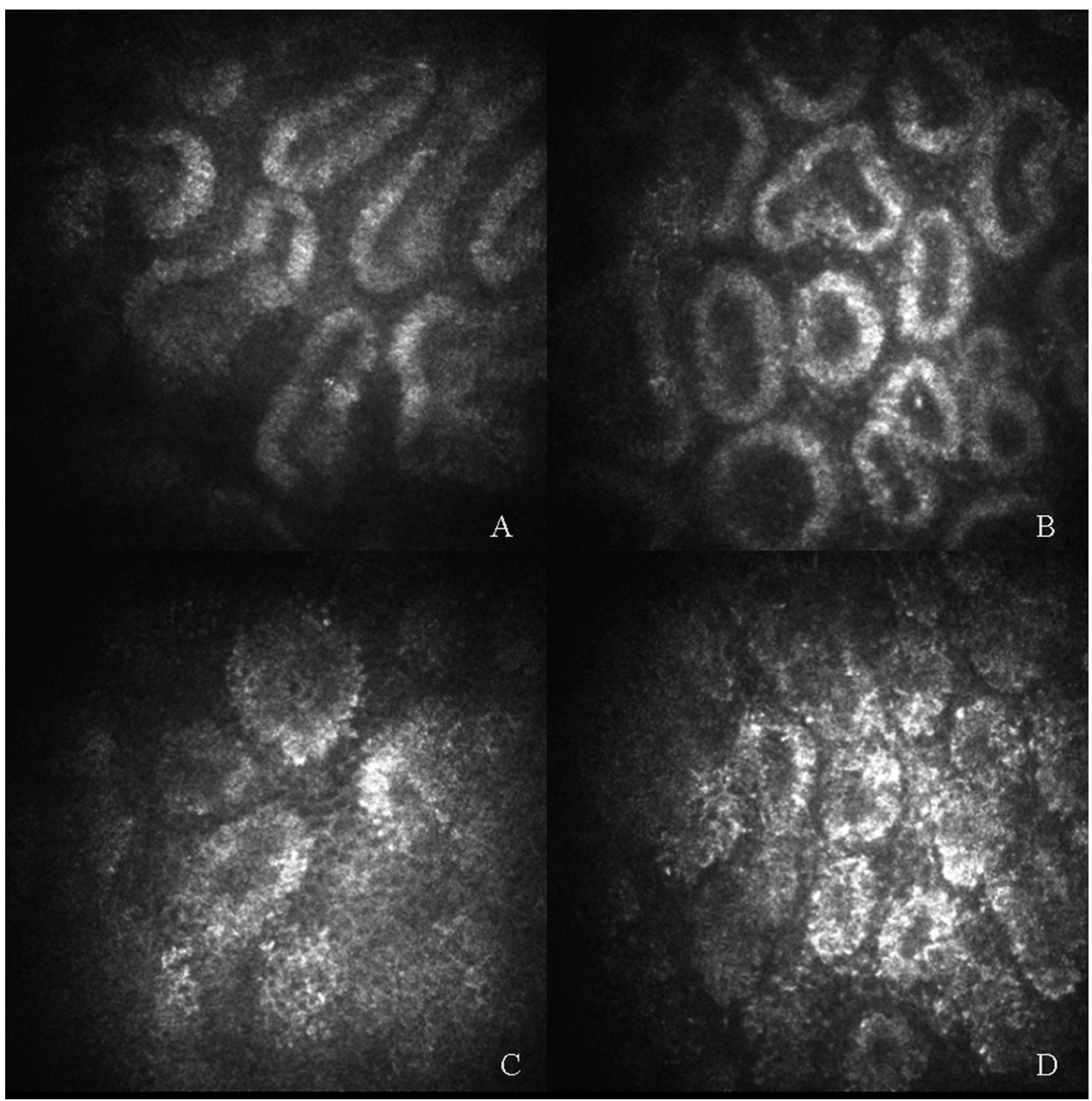

FIGURE 4. Inhomogeneous appearance of wall of acinar unit. Grading 1-4: (A) grade 1, absence of punctate reflecting elements; (B) grade 2, slight presence of punctate reflecting elements; (C) grade 3, greater presence of punctate reflecting elements; (D) grade 4, greatest presence of punctate reflecting elements.
(Fig. 2), and inhomogeneous appearance of interstices and walls of acinar units (Figs. 3 and 4). For the last three variables, we adopted three grading scales (1-4) assessed by comparison with the reference images (Figs. 2-4). Values obtained by the same investigator (SB) 1 month later and by a second independent investigator (MDC) were used to quantify intra- and interobserver agreement, respectively.

\section{Statistical Analysis}

All data are presented as mean \pm SD. For each variable, the Kruskal-Wallis test was applied to test the statistical differences between the four groups. The Mann-Whitney $U$ test was used as a post hoc test to determine the difference between individual pairs of groups. Correlations among variables were analyzed with Spearman's index of linear correlation.

TABLe 1. Clinical Data

\begin{tabular}{|c|c|c|c|c|c|c|c|}
\hline & OSDI & MG Dropout & BUT & Corneal Staining & $\begin{array}{l}\text { Conjunctival } \\
\text { Staining }\end{array}$ & Schirmer Test & $\begin{array}{c}\text { MG } \\
\text { Expressibility }\end{array}$ \\
\hline SSI & $26.5 \pm 12.8$ & $1.3 \pm 0.7$ & $3.7 \pm 1.8$ & $4.2 \pm 3.3$ & $2.7 \pm 0.9$ & $1.9 \pm 0.9$ & $1.7 \pm 0.9$ \\
\hline SSII & $18.2 \pm 6.9$ & $1.2 \pm 0.7$ & $5.1 \pm 2.9$ & $2.8 \pm 1.9$ & $1.6 \pm 0.5$ & $3 \pm 1.9$ & $1.7 \pm 0.9$ \\
\hline MGD & $13.3 \pm 9.8$ & $1.6 \pm 0.5$ & $5.4 \pm 1.7$ & $1.2 \pm 0.9$ & $1.2 \pm 0.9$ & $12.3 \pm 1.9$ & $2.5 \pm 0.6$ \\
\hline C & $4.9 \pm 3.4$ & $0.4 \pm 0.5$ & $9.2 \pm 3.1$ & n.s. & n.s. & $9.5 \pm 5.6$ & $0.4 \pm 0.5$ \\
\hline$P^{*}$ & $<0.001 \dagger$ & $<0.001 \neq$ & $<0.001 \S$ & $<0.001 \|$ & $<0.001$ व & $<0.001 \#$ & $<0.001^{* *}$ \\
\hline
\end{tabular}

* $P$ obtained by Kruskal-Wallis test; n.s., not significant.

† SSI vs. SSII: $P<0.01$; SSI vs. C: $P<0.001$; SSII vs. C: $P<0.001$; MGD vs. C: $P<0.01$; SSI vs. MGD: $P<0.001$; SSII vs. MGD: n.s. (Mann-Whitney $U$ test)

‡SSI vs. SSII: n.s.; SSI vs. C: $P<0.001$; SSII vs. C: $P<0.001$; MGD vs. C: $P<0.001$; SSI vs. MGD: $P<0.05$; SSII vs. MGD: $P<0.05$ (Mann-Whitney $U$ test)

『 SSI vs. SSII: n.s.; SSI vs. C: $P<0.001$; SSII vs. C: $P<0.001$; MGD vs. C: $P<0.001$; SSI vs. MGD: $P<0.01$; SSII vs. MGD: n.s. (Mann-Whitney $U$ test).

$\|$ SSI vs. SSII: n.s.; SSI vs. MGD: $P=0.001$; SSII vs. MGD: $P<0.01$ (Mann-Whitney $U$ test).

II SSI vs. SSII: $P<0.001$; SSI vs. MGD: $P<0.001$; SSII vs. MGD: n.s. (Mann-Whitney $U$ test).

\# SSI vs. SSII: n.s.; SSI vs. C: $P<0.001$; SSII vs. C: $P<0.001$; MGD vs. C: $P<0.001$; SSI vs. MGD: $P<0.001$; SSII vs. MGD: $P<0.001$ (Mann-Whitney $U$ test)

${ }^{* * *}$ SSI vs. SSII: n.s.; SSI vs. C: $P<0.001$; SSII vs. C: $P<0.001$; MGD vs. C: $P<0.001$; SSI vs. MGD: $P<0.001$; SSII vs. MGD: $P<0.001$ (Mann-Whitney $U$ test) 
TABLE 2. Cell Density of Superficial and Basal Epithelium of the Eyelid Margin

\begin{tabular}{lcc}
\hline & \multicolumn{2}{c}{ Cell Density $\left(\right.$ cell $\left./ \mathbf{m m}^{2}\right)$} \\
\cline { 2 - 3 } & Superficial Epithelium & Basal Epithelium \\
\hline SSI & $1350 \pm 226$ & $3351 \pm 217$ \\
SSII & $1286 \pm 277$ & $3987 \pm 285$ \\
MGD & $1278 \pm 290$ & $3890 \pm 342$ \\
C & $1473 \pm 458$ & $4446 \pm 619$ \\
$P^{*}$ & n.s. & $<0.001 \dagger$ \\
\hline
\end{tabular}

n.s., not significant.

* Obtained by Kruskal-Wallis test.

† SSI vs. SSII: $P<0.01$; SSI vs. C: $P<0.001$; SSII vs. C: $P<0.05$; MGD vs. C: $P<0.05$; SSI vs. MGD: $P<0.05$; SSII vs. MGD: n.s (Mann-Whitney $U$ test)

The minimum criterion for tests of significance was $P<0.05$. Statistical analysis was conducted using commercial software (SPSS for Windows, v. 12.0; SPSS Sciences, Chicago, IL).

\section{RESUlTS}

\section{Clinical Data}

All clinical parameters showed statistically significant differences among groups ( $P<0.001$, Kruskal-Wallis test). All comparisons among groups are shown in Table 1.

\section{Cell Density Data}

There were no significant differences in superficial epithelium cell density among groups. Basal epithelium cell density was significantly higher in the C group, compared with other groups (Table 2).

\section{MG Confocal Data}

MGs had significantly higher diameters and lower density in patients with MGD compared with other groups. SSI and SSII patients had significantly reduced MG orifice diameters (Table 3).

MGD patients showed significantly higher secretion reflectivity compared with other groups.

SSI and SSII patients' secretion reflectivity presented significant differences compared with MGD and C groups. SSI and

TABLe 3. MG Acinar Unit Density and MG Acinar and Glandular Orifice Diameters

\begin{tabular}{lccc}
\hline \multirow{2}{*}{ Acinar Unit } & & $\begin{array}{c}\text { Glandular } \\
\text { Orifices }\end{array}$ \\
\cline { 2 - 3 } & $\begin{array}{c}\text { Density } \\
\left(\text { units } / \mathbf{m m}^{2}\right)\end{array}$ & $\begin{array}{c}\text { Diameter } \\
(\boldsymbol{\mu m})\end{array}$ & $\begin{array}{c}\text { Diameter } \\
(\boldsymbol{\mu m})\end{array}$ \\
\hline SSI & $138 \pm 69$ & $53 \pm 31$ & $27.8 \pm 5.9$ \\
SSII & $97 \pm 43$ & $70 \pm 42$ & $20.6 \pm 5.1$ \\
MGD & $57 \pm 21$ & $106 \pm 41$ & $50.0 \pm 9.1$ \\
C & $110 \pm 31$ & $53 \pm 14$ & $34.7 \pm 4.3$ \\
$P^{*}$ & $<0.001 \dagger$ & $<0.001 \dagger$ & $<0.001 \neq$ \\
\hline
\end{tabular}

n.s., not significant.

* Obtained by Kruskal-Wallis test.

† SSI vs. SSII: n.s.; SSI vs. C: n.s.; SSII vs. C: n.s.; MGD vs. C: $P<$ 0.001 ; SSI vs. MGD: $P<0.001$; SSII vs. MGD: $P<0.001$ (Mann-Whitney $U$ test).

$\ddagger$ SSI vs. SSII: $P<0.05$; SSI vs. C: $P<0.05$; SSII vs. C: $P<0.001$; MGD vs. C: $P=0.001$; SSI vs. MGD: $P=0.001$; SSII vs. MGD: $P<0.001$ (Mann-Whitney $U$ test).
SSII patients showed higher inhomogeneous appearance of periglandular interstices than did other groups. MGD patients' interstices' inhomogeneous appearance was significantly higher than C but lower than SS patients. SS and MGD patients showed higher inhomogeneous appearance of the acinar wall compared with the C group (Table 4).

Secretion reflectivity, inhomogeneous appearance of periglandular interstice, and inhomogeneous appearance of the acinar wall showed substantial intra- and interobserver agreement ( $K=0.92$ and 0.80 , respectively), secretion reflectivity $(0.86$ and 0.78 , respectively), inhomogeneous appearance of periglandular interstice ( 0.82 and 0.78 , respectively), and inhomogeneous appearance of acinar wall.

In SS patients, the grade of inhomogeneous appearance of periglandular interstices and the acinar wall were significantly interrelated and correlated with corneal fluorescein staining ( $P \leq 0.01$, Spearman's correlation coefficient). Acinar density and diameter were strongly correlated between themselves $(P<0.001)$ and with BUT $(P<0.05)$.

\section{Discussion}

SS is a chronic autoimmune disorder characterized by dysfunction and destruction of exocrine glands resulting in an aqueous tear-deficient dry eye. ${ }^{5}$ Recent literature suggests that within SS there exists an organic hybrid form of aqueous-deficient dry eye (ADDE) - inflammatory infiltration of the lacrimal glandand evaporative dry eye (EDE)-MGD. ${ }^{16}$

LSCM has been recently applied to the armament of modalities used in the examination of MGs, providing a new noninvasive tool with which to study morphologic changes in MGs. ${ }^{12,14}$ LSCM is a technology that allows description and testing of the diagnostic values of acinar density and diameter, secretion reflectivity, and periglandular inflammation in MGD patients.

Previous studies ${ }^{12,14}$ described MGs in vivo confocal examinations performed through the tarsal conjunctiva of the inverted superior eyelid. A confocal examination needs a prolonged contact (a few minutes) between instrument and examined tissue, which can be uncomfortable for the patient during inverted superior eyelid tarsal conjunctiva evaluation. Moreover the instrument has a space fix orientation, so it requires the examined tissue to be positioned parallel to the polymethacrylate sterile cap (Tomo-Cap) face; this is

TABLE 4. Grade of Secretion Reflectivity and Inhomogeneous Appearance of Interstices and Wall of MG Acini

\begin{tabular}{lccc}
\hline Secretion & $\begin{array}{c}\text { Inhomogeneous } \\
\text { Reflectivity }\end{array}$ & $\begin{array}{c}\text { Appearance of } \\
\text { Interstice }\end{array}$ & $\begin{array}{c}\text { Inhomogeneous } \\
\text { Appearance of } \\
\text { Wall }\end{array}$ \\
\hline SSI & $1.7 \pm 0.6$ & $2.6 \pm 0.6$ & $3.0 \pm 1.0$ \\
SSII & $2.2 \pm 0.8$ & $2.6 \pm 0.6$ & $2.8 \pm 0.8$ \\
MGD & $3.3 \pm 0.7$ & $1.8 \pm 0.8$ & $2.5 \pm 0.9$ \\
C & $1.1 \pm 0.7$ & $1 \pm 0.3$ & $1.4 \pm 0.5$ \\
$P^{*}$ & $<0.001 \dagger$ & $<0.001 \neq$ & $<0.0015$ \\
\hline
\end{tabular}

n.s., not significant.

* Obtained by Kruskal-Wallis test.

† SSI vs. SSII: n.s.; SSI vs. C: $P<0.05$; SSII vs. C: $P<0.001$; MGD vs. C: $P<0.001$; SSI vs. MGD: $P<0.001$; SSII vs. MGD: $P<0.001$ (Mann-Whitney $U$ test)

‡ SSI vs. SSII: n.s.; SSI vs. C: $P<0.001$; SSII vs. C: $P<0.001 ;$ MGD vs. C: $P<0.001$; SSI vs. MGD: $P<0.01$; SSII vs. MGD: $P=0.001$ (Mann-Whitney $U$ test).

\ SSI vs. SSII: n.s.; SSI vs. C: $P<0.001$; SSII vs. C: $P<0.001$; MGD vs. C: $P<0.001$; SSI vs. MGD: n.s.; SSII vs. MGD: n.s. (Mann-Whitney $U$ test). 


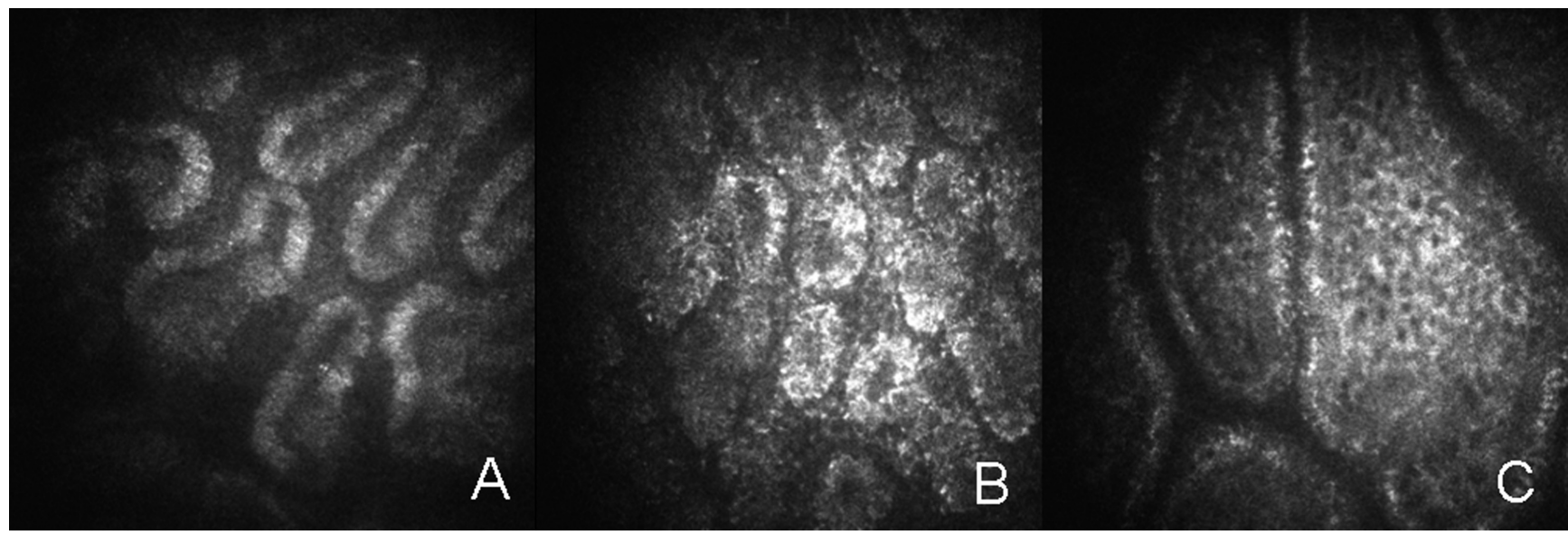

FIGURE 5. (A) MG in healthy subject; (B) MG in SS; (C) MG in MGD.

the reason why it is very difficult to evaluate the superior eyelid margin, whereas the lower one is easily positioned and inspected. By performing LSCM of the eyelid margin, we were able to visualize not only the MG acini but also mucocutaneous junction epithelia and glandular orifices. We believe that the chance of studying by in vivo microscopy the mucocutaneous junction is extremely interesting, not only because of potential clinical information derivable in the different pathologies involving this structure, but also because of its role, recently emphasized in the literature, ${ }^{17,18}$ as a potential focal point of mucosal epithelial cell differentiation activities.

We performed LSCM of MGs in SS patients, comparing characteristics with MGD patients and healthy subjects.

The basal mucocutaneous epithelium showed a lower cell density in SS and MGD patients than in the control group, suggesting that mechanical and inflammatory damage cannot be compensated by proliferative changes of the basal epithelium, as happens, for example, in the cornea. ${ }^{19,20}$

MGD patients were characterized by extensive MG dropout, MG secretion that could not be expressed, tear film instability, and ocular surface epithelial damage. According to recent literature, ${ }^{12-14}$ LSCM in these patients showed increased diameters of acinar units and orifices and highreflective secretion. These alterations may be due to qualitative changes of the MG secretion (inspissation, increased viscosity, and build-up) and to consequent MG obstruction.

Patients with SS showed less acinar dilatation, lower secretion reflectivity, and decreased orifice diameters, suggesting a minor role for the obstructive pathogenetic mechanism.

We interpreted the inhomogeneous appearance of the periglandular interstices as a confocal sign of eyelid margin and tarsal inflammation. Previous studies reported quantitative data regarding periglandular inflammatory dendritic cell density. ${ }^{12,14}$ In our hands, it was too difficult to identify individual cells. Furthermore, the dendritic shape was often unrecognizable. Thus, we chose to perform a semiquantitative analysis (1-4 grading) of the punctiform elements viewable in the interstices. This inflammatory sign was significantly increased in all pathologic groups but was highest in the SS group.

The inhomogeneous appearance of the acinar wall was significantly increased in all pathologic groups, with no differences between SS and MGD patients. This confocal feature is most likely interpretable as signifying inflammatory processes and changes in MG secretion characteristics.
In conclusion, LSCM offers new opportunities for in vivo noninvasive histopathological evaluations of the ocular surface. Its application to MGs showed discernible patterns of MG abnormalities in SS and MGD (Fig. 5), not easily distinguishable by usual clinical exams, such as meibography and MG secretion expression. In SS patients we observed a prevalence of confocal signs of inflammation, without dilatative morphologic changes, while in MGD patients we found a prevalence of signs of glandular obstruction and distension.

Further studies regarding confocal features of MGs in various types of ocular surface diseases could lead to a new morpho-functional classification of $\mathrm{MG}$ alterations and to additional therapeutic modalities.

\section{Acknowledgments}

The authors thank Kathryn S. Pokorny (IOVS Volunteer Editor) for her kind assistance with English grammar and usage.

\section{References}

1. Theander E, Jacobsson LT. Relationship of Sjögren's Syndrome to other connective tissue and autoimmune disorders. Rbeum Dis Clin North Am. 2008;34:935-947.

2. Moutsopoulos HM. Sjögren's Syndrome: autoimmune epitheliitis Clin Immunol Immunopatbol. 1994;72:162-165.

3. Mitsias DI, Kapsogeorgou EK, Moutsopoulos HM. Sjögren's Syndrome: why autoimmune epitheliitis? Oral Dis. 2006;12:523532.

4. Adamson T, Fox R, Frisman D, et al. Immunohistological analysis of lymphoid infiltrates in primary Sjögren's Syndrome using monoclonal antibodies. J Immunol. 1983;130:203.

5. The definition and classification of dry eye disease: report of the Definition and Classification Subcommittee of the International Dry Eye Workshop (2007). Ocul Surf. 2007;5(2):75-92.

6. Mathers WD. Ocular evaporation in meibomian gland dysfunction and dry eye. Opbthalmology. 1998;100:347-351.

7. Goto E, Endo K, Suzuki A, et al. Tear evaporation dynamics in normal subjects and subjects with obstructive meibomian gland dysfunction. Invest Opbthalmol Vis Sci. 2003;44:533-539.

8. Shimazaki J, Sakata M, Tsubota K. Ocular surface changes and discomfort in patients with meibomian gland dysfunction. Arch Ophthalmol. 1995;113:1266-1270.

9. Goto E, Matsumoto Y, Kamoi M, Endo K, et al. Tear evaporation rates in Sjögren Syndrome and non-Sjögren dry eye patients. Am J Opbthalmol. 2007;144:81-85.

10. Shimazaky J, Goto E, Masafumi O, Shimmura S, Tsubota K. Meibomian gland dysfunction in patients with Sjögren Syndrome. $O p b$ thalmology. 1998;105:1485-1488. 
11. Villani E, Galimberti D, Viola F, Ratiglia R. In vivo confocal microscopy of the ocular surface. Am J Opbthalmol. 2010;149: 689-690.

12. Matsumoto Y, Sato EA, Dogru M, Tsubota K. The application of in vivo laser confocal microscopy to the diagnosis and evaluation of meibomian gland dysfunction. Mol Vis. 2008;14:1263-1271.

13. Matsumoto Y, Shigeno Y, Sato E, Tsubota K, et al. The evaluation of the treatment response in obstructive meibomian gland disease by in vivo laser confocal microscopy. Arch Exp Opbthalomol. 2009;247:821-829.

14. Ibrahim OM, Matsumoto Y, Dogru M, et al. The efficacy, sensitivity, and specificity of in vivo laser confocal microscopy in the diagnosis of meibomian gland dysfunction. Opbthalmology. 2010; 117:665-672.

15. Vitali C, Bombardieri S, Jonsson R, et al. Classification criteria for Sjögren's Syndrome: a revised version of the European criteria proposed by the American-European Consensus Group. Ann Rbeum Dis. 2002;61:554-558.

16. Bron A, Yokoy N, Gaffney E, Tiffany JM. Predicted phenotypes of dry eye: proposed consequences of its natural history. Clin Sci. 2009;7:78-92.

17. Liu S, Li J, Tan DT, Beuerman RW. The eyelid margin: a transitional zone for 2 epithelial phenotypes. Arch Ophthalmol. 2007;125:523-532.

18. Riau AK, Barathi VA, Beuerman RW. Mucocutaneous junction of eyelid and lip: a study of the transition zone using epithelial cell markers. Curr Eye Res. 2008;33:912-922.

19. Fabiani C, Barabino S, Rashid S, Dana M. Corneal epithelial proliferation and thickness in a mouse model of dry eye. Exp Eye Res. 2009;89:166-171.

20. Villani E, Galimberti D, Viola F, Mapelli C, Del Papa N, Ratiglia R. Corneal involvement in rheumatoid arthritis: an in vivo confocal study. Invest Ophthalmol Vis Sci. 2008;49:560-564. 